\title{
TEMA 5-2016: Insuficiencia Cardiaca con Fracción de Eyección Conservada
}

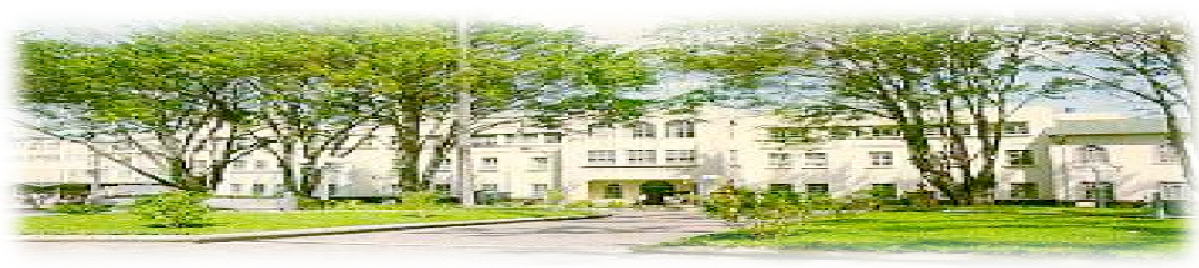

Hospital San Juan de Dios, San José, Costa Rica. Fundado en 1845
Recibido:

Aceptado:
$16 / 11 / 2015$

$15 / 12 / 2015$

\author{
Enrique Pacheco Ovares \\ Stanley Grant Villegas ${ }^{2}$
}

\footnotetext{
${ }^{1}$ Médico General, correo electrónico: cripacheco_17@hotmail.com

${ }^{2}$ Médico Especialista en Medicina Interna, Caja Costarricense de Seguro Social
}

\section{RESUMEN}

La creciente tasa de insuficiencia cardíaca (IC ) con fracción de eyección conservada ha recibido más atención por parte de la sociedad médica internacional. La alta prevalencia de enfermedades cardiovasculares ha hecho de la IC un diagnóstico común en nuestras sociedades. Es importante diferenciar síntomas tales como edema o falta de respiración por otras causas no cardiovasculares tales como la EPOC, la obesidad, anemia , medicamentos . Las conclusiones de los altos niveles de los biomarcadores específicos, los síntomas relacionados con la IC, y la estructura cardiovascular y alteraciones funcionales son la piedra angular para el diagnóstico . Desafortunadamente no hay pruebas directas limitadas para apoyar un régimen específico de medicamentos para el tratamiento de la IC con fracción de eyección conservada .

\section{PALABRAS CLAVE}

Insuficiencia cardiaca con fracción de eyección conservada, insuficiencia cardiaca, tratamiento, pronóstico, cardiología.

\section{ABSTRACT}

The increasing rate of heart failure (HF) with preserved ejection fraction has received more attention from the international medical society. The high prevalence of cardiovascular diseases has made of HF a common diagnosis in our societies. It is important to differentiate symptoms such as edema or shortness of breathing from other non cardiovascular causes such as COPD, obesity, anemia, medications. The findings of high levels of specific biomarkers, symptoms related to HF, and cardiovascular structure and functional abnormalities are the cornerstone for the diagnosis. Unfortunately there is limited 
direct evidence to support a specific drug regimen to treat HF with preserved ejection fraction.

\section{KEY WORDS}

heart failure with preserved ejection fraction, heart failure, treatment, prognosis, cardiology.

\section{INTRODUCCIÓN}

La insuficiencia cardiaca (IC) es un síndrome clínico donde se encuentra alterada la función $\mathrm{y} / \mathrm{o}$ estructura miocárdica, conllevando a un detrimento de la calidad de vida del paciente y de su esperanza de vida ${ }^{(1)}$. Dentro de las diferentes clasificaciones de IC, se puede tomar como parámetro la condición de la fracción de eyección del ventrículo izquierdo (FEVI), donde se puede considerar dos importantes categorías: la IC con disminución de la fracción de eyección (FEVI $<40 \%$ ), conservación de la fracción de eyección (FEVI $>40-50 \%$ ) o bien condiciones de alto gasto cardiaco.

La IC con FEVI conservada es un síndrome complejo caracterizado por signos y síntomas de IC y una normal o casi normal fracción de eyección del ventrículo izquierdo (VI). Criterios diagnósticos más específicos han evolucionado en el tiempo e incluyen signos/síntomas de IC, evidencia objetiva de disfunción diastólica, alteración del llenado ventricular, daño cardiovascular estructural y niveles elevados de péptidos natriuréticos ${ }^{(2)}$.

En el presente artículo se considerarán los aspectos pertinentes a la IC con fracción de eyección conservada.

\section{Epidemiología}

Se considera que cerca de 20 millones de personas alrededor del mundo presentan IC, con una prevalencia general en la población adulta en países industrializados en $2 \%$, donde se presenta un crecimiento exponencial con la edad, llegando a afectar entre un $6-10 \%$ de la población mayor a los 65 años de edad (3). Presenta una predilección por el sexo masculino que el femenino, sin embargo interesantemente la prevalencia es mayor en mujeres debido a su mayor esperanza de vida en comparación con el sexo masculino. En relación a la IC con FEVI conservada propiamente, cerca de un $50 \%$ de los pacientes que son hospitalizados por presentar síntomas de insufi- ciencia cardiaca o bien son diagnosticados con IC; presentan IC con FEVI conservada. ${ }^{(4,5)}$

Se considera que el riesgo de presentar IC en Estados Unidos y Europa es de casi uno en cinco a los 40 años de edad.

\section{DISCUSIÓN}

\section{Generalidades.}

Dentro de las principales causas de IC con fracción de eyección conservada se encuentra la hipertrofia patológica que puede ser tanto primaria (miocardiopatía hipertrófica) o bien secundaria como ocurre en casos de hipertensión arterial, envejecimiento, miocardiopatía restrictiva asociada a trastornos infiltrativos como lo son la amiloidosis o sarcoidosis o bien enfermedades por almacenamiento como la hemocromatosis. Otras causas lo son la fibrosis y trastornos endomiocárdicos. Es importante destacar que indistintamente si el paciente presente un FEVI conservada o disminuida, presentaran el síndrome clínico como tal y compartiendo muchas características similares a lo largo del espectro de la $\mathrm{FE}$, incluyendo una dinámica de llenado anormal del ventrículo izquierdo (VI), presión diastólica elevada en el VI, disfunción sistólica y diastólica del VI, activación neurohormonal, alteración de la tolerancia al ejercicio, aumento en la frecuencia de ingresos hospitalarios y reducción de la supervivencia ${ }^{(6,7)}$

La mortalidad que presentan los pacientes con IC con FEVI conservada es alta, con una tasa de mortalidad a 5 años de aproximadamente el $60 \%$, con una morbilidad costosa llevando a una tasa de hospitalización en 6 meses del $50 \%$ y síntomas debilitantes con un promedio de máximo consumo de oxigeno miocárdico (MVO2) de 14 $\mathrm{ml} / \mathrm{g} / \mathrm{min}$. ${ }^{(7)}$

En contraste con aquellos pacientes que presentan IC con FEVI disminuida, los pacientes con IC con FEVI conservada presentan mayor edad y más probabilidad de ser mujeres, sin embargos la IC con FEVI conservada afecta tanto a mujeres como hombres en las décadas quinta a novena de la vida. El antecedente clínico más frecuente para pacientes con IC con FEVI conservada es la hipertensión sistólica, que esta presenta en cerca del $85 \%$ de los pacientes, mientras que la cardiopatía isquémica es mucho menos habitual que en la IC con FEVI reducida. Otras diferencias que se reconocen son que en pacientes con IC con 
FEVI conservado el volumen telediastólico del VI normal y normal o casi normal FEVI y volumen telediastólico, frecuentemente muestran remodelado concéntrico de la cámara del VI y/o de los miocitos cardiacos.

La principal causa de mortalidad en pacientes con IC con FEVI conservada son de naturaleza cardiovascular en un $70 \%$ de los casos, con un $20 \%$ debido a insuficiencia cardiaca y $35 \%$ por muerte súbita. Esta distribución muestra similitud en los casos con FEVI reducida ${ }^{(8)}$. La incidencia de muertes no cardiovasculares es significativamente mayor para la IC con FEVI conservada $(30 \%)$ que con FEVI reducida $(15 \%)$.

La tasa de supervivencia a 5 años para todos los pacientes con insuficiencia cardiaca, independientemente de de la FEVI, es inferior a 50\%. Aunque la supervivencia ha mejorad a lo largo del tiempo para los pacientes con FEVI reducida, no ha cambiado para los pacientes con FEVI conservada ${ }^{(9)}$.

\section{Fisiopatología.}

Entendiendo la IC como un afectación en la estructura $\mathrm{y} / \mathrm{o}$ función miocárdica, debemos entender estos dos principios para poder comprender los mecanismos o factores que van a contribuir en la fisiopatología de la IC con FEVI conservada. Contemplando alteraciones estructurales, se presentan de forma primordial el remodelado concéntrico del miocardio o la hipertrofia del VI. Con respecto a las afectaciones de la función del VI se encuentra la disfunción diastólica secundaria a una alteración en la relajación, con disminución de recuperación, llenado anormal, distensibilidad disminuida y aumento de la presión diastólica. En caso de presentarse disfunción sistólica se ha detectado un acortamiento anormal de la zona media de la pared y del eje largo con disminución de la torsión. A estos fenómenos se pueden asociar un aumento de la carga hemodinámica con aumento de la poscarga y de la carga de llenado, aumento de volumen y rigidez de la aurícula izquierda, disminución de la función de reserva de la aurícula izquierda, de la función pasiva de conducto y de la función de bomba activa ${ }^{(\mathbf{1 0})}$

En casos de pacientes que hayan presentado isquemia, es también un mecanismo fisiopatológico importante, ya que la enfermedad subendocárdica y microvascular conllevan a una alteración de la reserva de flujo coronario, pulmonar y periférico. Otros mecanismos como alteracio- nes del ritmo (incompetencia cronotrópica, fibrilación auricular, taquicardia supraventricular, etc).

En corazones normales a lo largo de un rango de frecuencias cardiacas normales, la relajación y recuperación resultan adecuadas para permitir que las presiones de la aurícula izquierda (AI) se mantengan normales. De igual forma el efecto de catecolaminas potencian la relajación y recuperación durante el ejercicio, logra disminuir las presiones en el VI en la diástole precoz, aumentando de esta forma, el gradiente de presión de la AI al VI sin elevar las presiones de la AI, así como potencia el llenado durante el ejercicio.

En pacientes que presentan IC con FEVI conservada, la relajación y recuperación son anormales en reposo y no se potencian de forma adecuada al realizar actividades que aumenten la frecuencia cardiaca o bien durante el ejercicio, de manera que no favorecen el gradiente entre la AI y VI. La única forma de lograr mantener el llenado es elevando la presión de la AI, la cual debe en este momento "impulsar" la sangre hacia el ventrículo izquierdo para mantener un adecuado gasto cardiaco. Dentro de los mecanismos compensadores que forman parte de la IC se encuentran la activación de los sistemas de reninaangiotensina-aldosterona, del sistema nervioso adrenérgico y el incremento de la contractilidad miocárdica. Es importante mencionar que además hay activación de la familia de vasodilatadores, lo que incluye a los péptidos auricular y encefálico natriuréticos (ANP y BNP respectivamente), que evitan la vasoconstricción periférica excesiva.

Propiamente en los miocitos cardiacos se da una alteración en la homeostasis del calcio, aumentando el calcio diastólico o disminuyendo el índice de recuperación del calcio, llevando a relajación alterada o incompleta.

En casos de isquemia la cantidad de ATP se encuentra reducida, interfiriendo con el proceso de relajación miocárdica. Se debe recordar que el proceso de relajación miocárdica es un proceso dependiente de ATP, el cual está regulado por la captación de calcio citoplasmático o en el retículo sarcoplásmico por acción de SERCA2A y por la salida de calcio intracelular por acción de las bombas del sarcolema. También puede ocurrir retraso en el llenado del VI por disminución de la distensibilidad de dicho ventrículo.

Esto presenta una pérdida de miocitos por necrosis, apoptosis o autofagia, alteración de la matriz 
extracelular con degradación de la misma y fibrosis miocárdica (recordando que el tejido fibrótico no es funcional, por lo tanto el tejido miocárdico funcional está siendo reemplazado por tejido no funcional, conllevando a disminución de la función cardiaca). Estos cambios miocárdicos obedecen a la hipertrofia, una desensibilización $\beta$ - adrenérgica y alteración en el acoplamiento de excitación- contracción miocárdica. De forma macroanatómica estos cambios se traducen en dilatación ventricular izquierda, aumento de la esfericidad del ventrículo izquierdo, adelgazamiento de la pared del VI e insuficiencia de la válvula mitral. La alteración estructural y funcional que se presentan en pacientes con IC correlaciona directamente los signos y síntomas propios de la enfermedad.

\section{Clínica y Diagnóstico.}

Para el diagnóstico de IC con FEVI conservada se requiere de un paciente que presente signos y síntomas de IC, con una FEVI superior al 50\% y evidencia objetiva de disfunción cardiaca. Entre las manifestaciones más frecuentes se encuentra la intolerancia al ejercicio, disnea asociada a esfuerzos, ortopnea, disnea paroxística nocturna, además periféricos y congestión pulmonar aparente en las radiografías. Se debe destacar que aunque se ha descrito que un pulso apical del VI desplazado y un pulso alternante están presentes solo en la IC con FEVI reducida, ninguna característica clínica (síntomas, signos o radiografías torácicas) pueden ser utilizadas para diferencias una IC con FEVI reducida o conservada ${ }^{(10)}$.

De manera característica es necesario mencionar que muchos síntomas de IC no necesariamente se deban a IC; por ejemplo la disnea que puede presentar un paciente se pueden deber a enfermedad pulmonar crónica, obesidad, anemia $u$ otra condición médica, de igual forma los edemas podálicos se pueden asociar a uso de medicamentos vasodilatadores como calcioantagonistas, obesidad mórbida o insuficiencia venosa. De esta forma la única forma de diagnosticar de forma certera una IC es con una adecuada medición de la función cardiovascular o bien alteraciones estructurales que puedan traducirse en una patología cardiovascular subyacente como causal de los síntomas que presenta el paciente. En pacientes con IC con FEVI conservada se supone un reto dado que la FEVI se encuentra normal y el volumen telediastólico no se encuentra aumen- tado, de manera que es necesario documentar biomarcadores elevados para concretar el diagnostico (como BNP), una función diastólica anormal del VI o un aumento de la AI para el diagnóstico. Desde luego, el diagnostico también supone descartar otras causas no cardiacas que puedan asociar los signos y síntomas del paciente. Los biomarcadores más adecuados son los péptidos natriuréticos, BNP y N-terminal del pro-BNP (NT pro-BNP), donde ciertamente los niveles de estos marcadores se encuentran elevados en pacientes con IC con FEVI conservada, no son tan elevados como en pacientes con FEVI reducida y estos niveles se encuentran directamente relacionados con la presión de llenado diastólico del VI y con el estrés telediastólico en la pared.

\section{Criterios Diagnósticos:}

Evidencia clínica de IC

- Presentación clínica clara de IC o

- Criterios de Framingham o

- Criterios Boston

Pruebas de laboratorio

- $\quad$ Plasma BNP $>100 \mathrm{pg} / \mathrm{ml}$, NT pro-BNP $>800$ o

- Radiografía de tórax o

- Pruebas cardiopulmonares de ejercicio

FEVI "conservada" $>50 \%$, con VTD VI normal $(<97 \mathrm{ml} / \mathrm{m} 2)$

Evidencia adicional de apoyo adicional: Hipertrofia ventrículo izquierdo concéntrica o remodelado concéntrico, crecimiento de la AI (sin fibrilación auricular) y evidencia de eco-Doppler o cateterismo de disfunción diastólica

Se debe excluir: Enfermedad no Miocárdica

\section{Tratamiento}

El tratamiento de la IC con FEVI conservada, se encuentra firmemente asociada al tratamiento de sus mismas condiciones como la hipertensión arterial y sus síntomas como en el caso de edema. De acuerdo a los lineamientos de la ACC/AHA 2013 sobre IC se incluyen las siguientes dos normativas como dos recomendaciones fuertes:

1. Hipertensión sistólica y diastólica se debe controlar de acuerdo a las guías publicadas para prevenir la morbilidad.

2. Los diuréticos se deben utilizar para aliviar los síntomas debidos a sobrecarga de volumen. 
El tratamiento de la IC con FEVI conservada difiere de la IC con FEVI reducida, donde los resultados de los estudios clínicos han demostrado que mientras los antagonistas neurohumorales como los beta bloqueadores, inhibidores de la enzima convertidora de angiotensina y bloqueadores del receptor de angiotensina, así como la resincronización cardiaca son efectivas en IC con FEVI reducida, estas terapias no disminuyen la morbilidad ni mortalidad en pacientes con IC con FEVI conservada. Los estudios sugieren que hay diferencias fundamentales en la fisiopatología subyacente en IC con FEVI conservada vs reducida. Todas las terapias que mejoran la mortalidad en pacientes con IC con FEVI reducida, también revierte la dilatación del VI. Pero dado que los pacientes con FEVI conservada presentan mínima o ninguna dilatación del VI, puede no sorprender que estos agentes no sean tan efectivos como para FEVI reducida ${ }^{(11)}$.

Hipertensión: La disminución de la presión arterial reduce la tasa subsecuente de desarrollo de IC en muchos estudios aleatorizados sobre hipertensión. La escogencia del antihipertensivo va de la mano con la individualización terapéutica; si el paciente asocia diabetes mellitus o EPOC. En un análisis hecho por ALLHAT, se demostró que clortalidona reduce la incidencia de IC con FEVI conservada, en comparación con amlodipina, lisinopril y doxazosina; mientras que ambos lisinopril y clortalidona fueren efectivos en reducir la incidencia de IC con FEVI reducida. (12)

La regresión de la hipertrofia del VI es una meta terapéutica importante, ya que mejora la función diastólica. En un meta análisis se evaluaron antihipertensivos y su eficacia relativa para revertir la hipertrofia del VI en pacientes con hipertensión, donde 80 estudios que incluyeron 146 tratamientos activos y 17 placebos, fueron evaluados. Luego de ajustes estadísticos por duración terapéutica y grado de la disminución en la hipertensión, las reducciones relativas de la masa del VI fueron:

\begin{tabular}{|c|c|}
\hline ARA II & $13 \%$ \\
\hline Calcio antagonistas & $11 \%$ \\
\hline IECA & $10 \%$ \\
\hline Diuréticos & $8 \%$ \\
\hline Beta bloqueadores & $6 \%$ \\
\hline
\end{tabular}

Pareciera que los ARA II, calcio antagonistas e IECA producen una mayor regresión que los beta bloqueadores. La importancia clínica de esta diferencia es incierta desde que aun no hay evidencia de que exista una mayor regresión de la hipertrofia del VI asociada a una mejor sobrevida a largo plazo, siendo otros factores los determinantes para la elección e individualización terapéutica del paciente con IC.

Fibrilación Atrial: es común en pacientes con FEVI conservada, se ha identificado que cerca de $2 / 3$ de los pacientes presentan fibrilación atrial y su presencia aumenta la morbilidad y mortalidad. En estos pacientes la restauración y mantenimiento del ritmo sinusal es preferido, dado que en pacientes con IC con FEVI conservada, la taquicardia disminuye el tiempo de diástole y dado que el llenado ventricular ocurre en la diástole tardía, esta es mucho más dependiente de la contracción atrial que en corazones normales. Los beta bloqueadores y calcio antagonistas son el tratamiento de primera línea, dejando a la digoxina más para pacientes con FEVI reducida. (13)

Enfermedad Coronaria: La presencia de enfermedad coronaria es un predictor independiente en el aumento de la mortalidad, junto con un mayor deterioro de la función sistólica del VI a lo largo del tiempo. Pacientes con FEVI conservada y síntomas y signos de isquemia son tratados con la terapia estandarizada incluyendo beta bloqueadores y calcio antagonistas. Nitratos son prescritos frecuentemente, pero la reducción de la precarga pueden conllevar a hipotensión en algunos pacientes. ${ }^{(14)}$

Hiperlipidemia: Se recomienda su tratamiento tanto para prevención primaria como secundaria. Dos estudios randomizados encontraron que las estatinas no tienen efecto beneficioso en pacientes con FEVI reducida, de todas maneras información observacional sugiere que las estatinas pueden ser beneficiosas en pacientes con FEVI conservada. Se recomienda su uso en pacientes con FEVI conservada que cumplan criterios para su uso.

En pacientes que presenten IC con FEVI conservada (incluyendo aquellos con BNP elevados) y en los cuales se puede monitorizar cuidadosamente los niveles séricos de potasio y su función renal, se recomienda el tratamiento con antagonistas del receptor mineralocorticoide. El inicio del tratamiento con espirinolactona debe ser con un potasio menor a $5.0 \mathrm{mEq} / \mathrm{L}$ y una TFG estimada mayor o igual a $30 \mathrm{ml} / \mathrm{min}$ por $1.73 \mathrm{~m} 2$.

El uso de diuréticos debe ser cuidadoso, siempre evitando una reducción excesiva de la precarga e 
hipotensión. El uso de estos medicamentos resulto ser beneficioso en el estudio CHAMPION, donde las decisiones terapéuticas basadas en la medición de la presión pulmonar arterial se asoció con una significante reducción en las hospitalizaciones por IC. ${ }^{(15)}$

\section{CONCLUSIONES}

- La diferencia fisiopatológica entre la IC con FEVI reducida vs conservada, hace que la eficacia terapéutica varia considerablemente.

- La prevalencia de la IC con FEVI conservada tiende a ir en aumento, de forma exponencial con la edad y debido a la cada vez mayor incidencia de patologías cardiovasculares, se prevé que esta enfermedad aumente considerablemente

- La meta terapéutica principal es la reducción en el remodelado ventricular izquierdo, que es el eje principal del cual derivan los signos y síntomas de la IC

- Existe lamentablemente, una evidencia directa muy limitada para apoyar el uso de un tratamiento en específico en pacientes con IC con FEVI conservada.

- El entrenamiento físico ha demostrado ser la única intervención que mejora la capacidad al ejercicio y la calidad de vida en pacientes con FEVI conservada.

\section{BIBLIOGRAFÍA}

1. 3. Dan L Longo Dennis L Kasper et al: Harrison Principios de Medicina Interna. Editorial McGraw Hill. Decimoctava edición, 2012. Vol 2 1901-1904.

2. Senni M J Paulus W Gavazzi A et al: New Strategies for heart failure with preserved ejection fraction: the importance of targeted therapies for heart failure phenotypes, European Society of Cardiology. European Heart Journal 35, 2797-2811, 2014.

3. Owan TE Hodge DO Herges RM et al: Trends in prevalence and outcome of heart failure with preserverd ejection fraction, New England Journal of Medicine 355:251, 2006.

4. Kitzman DW Little WC: Left ventricle diastolic dysfunction and prognosis, Circulation 125: 743, 2012.

5. Lam CSP Roger VL Rodeheffer RJ et al: Cardiac structure and ventricular-vascular function in persons with heart failure and preserved ejection fraction: From Olmstead County, Minnesota. Circulation 115:1982, 2007.

6. Little WC Zile MR: HFPEF: Cardiovascular abnormalities not just co-morbidities, Circ Heart Fail 5:669, 2012.

7. Little WC Zile MR Klein A et al: Effect of losartan and hydrochlorothiazide on exercise tolerance in extertional hypertension and left ventricular diastolic dysfunction, American Journal of Cardiology 98:383, 2006.

8. Zile MR Gaasch WH Anand IS et al: Mode of death in patients with heart failure and preserved ejection fraction: Results from the Irbesartan in Heart Failure with Preserved Ejection fraction Study (I-Preserve) trial, Circulation 121:1393, 2010.

9. Douglas L Mann P Zipes Petter Libby et al: Braunwald's Heart Disease. A textbook of Cardiovascular Medicine. El Sevier. Décima Edición 2015, pag 557

10. A Borlaug S Colucci B Yeon: Treatment and prognosis of heart failure with preserved ejection fraction. Up to date, 2015.

11. Aurigemma GP Gaasch WH. Clinical Practice. Diastolic heart failure. New England Med 2004; 351:1097

12. Sharma K Kass DA. Heart failure with preserved ejection graction: mechanisms, clinical features, and therapies. Circ Res 2014; 115:79

13. Yancy CW Jessup M Bozkurt, et al: 2013 ACCF/AHA guideline for the management of heart failure: a report of the American College of Cardiology Foundation/ American Heart Association Task Force on Practice Guidelines. $\mathrm{J}$ Am Coll Cardiol 2013; 62: e147

14. Owan TE Hodge DO Herges RM et al: Trends in prevalence and outcome of heart failure with preserved ejection fraction. $\mathrm{N}$ Engl $\mathrm{J}$ Med 2006

\section{CONFLICTOS DE INTERES}

Los autores declaran que no existe ningún conflicto de interés. 